\title{
Serine/Threonine Protein Phosphatase 2B Catalytic Subunit Gamma Isoform
}

National Cancer Institute

\section{Source}

National Cancer Institute. Serine/Threonine Protein Phosphatase 2B Catalytic Subunit

Gamma Isoform. NCI Thesaurus. Code C38527.

Serine/threonine-protein phosphatase 2B catalytic subunit gamma isoform (512 aa, $\sim 58$

$\mathrm{kDa}$ ) is encoded by the human PPP3CC gene. This protein is involved in protein

dephosphorylation, calmodulin binding, and apoptotic signaling. 